\author{
INVESTIGATING THE RATIONALE FOR EXTENDING CT KUB TO INCLUDE THE \\ CHEST FOR SUSPECTED RENAL COLIC PATIENTS DURING THE COVID-19 \\ PANDEMIC \\ Y Abdelmotagly ${ }^{1,3}$, M Noureldin $^{1,2}$, L Paramore ${ }^{1}$, G Rajkumar $^{1}$, G Ubhayaka $^{1}$, T Nedas $^{1}$, R Hindley ${ }^{1}$, \\ A Emara ${ }^{1,2}$ \\ ${ }^{1}$ Hampshire Hospitals NHS Foundation Trust, United Kingdom. \\ ${ }^{2}$ Urology Department, Ain Shams University, Egypt. \\ ${ }^{3}$ Urology Department, Misr University for Science and Technology, Egypt.
}

Correspondence: yehiazakyuk@gmail.com

Submitted: July 3, 2020. Accepted: July 7, 2020. Published: August 21, 2020.

\title{
Background and Objective
}

Following on from the Royal College of Surgeons (RCS) recommendation of 5th of April 2020 to perform computed tomography (CT) scan of the chest in patients presenting with an abdominal pain emergencies and undergoing an abdominal CT; comparison was made between a cohort of patients with a RCS-COVID recommended scan and a similar group of patients in the pre-COVID era.

To evaluate the value of extending CT-KUB scan to include the chest area, in patients presenting to the emergency department with acute renal colic during the COVID-19 pandemic.

\section{Material and Methods}

Retrospective data included; initial presentation, COVID-19-related symptoms, dose length product (DLP), scan extension, Ground glass appearance (GGO), Wuhan corona virus swab polymerase chain reaction (PCR) test.

\section{Results}

A total of 100 patients underwent CT KUB in April 2020 (Group 1) with a similar number of patients from the pre-COVID-19 pandemic randomly selected from August to October 2019 (Group 2). Patients age ranged from 15 to 91 years with a median of 48 years in Group 1, whilst in Group 2 the range was 19 to 85 years with a median of 50 years. All patients in both groups initially presented with renal colic. No COVID-19-related respiratory manifestations were recorded.

Nine patients from Group 1 had GGO identified in their chest CT with all of them returning with negative COVID-19 swabs. Interestingly there was almost the same number of stones diagnosed 49\% (49/ $100)$ in Group 1 patients compared with 50\% (23/5150/100) from Group 2.

\section{Conclusions}

Despite the RCS COVID CT scan recommendations, our study has demonstrated no significant additional value of extending the CT-KUB to include the chest area in renal colic patients with no respiratory manifestations. Further studies are recommended in order to validate these results.

Keywords: COVID-19; SARS-CoV-2; Urology; CT KUB, chest CT 
Following the outbreak of coronavirus disease 2019 (COVID-19) caused by a novel strain of coronavirus severe acute respiratory syndrome coronavirus 2 (SARS-CoV-2 originating from Wuhan, China in December 2019. The virus has spread around the globe and has been declared a global pandemic by the World Health Organization in February 2020. By end of April 2020, there were 3,136,507 confirmed cases of COVID-19 affecting 210 countries and territories around the world. ${ }^{1,2}$

Understanding the clinical symptoms of COVID-19 is important, although they can be nonspecific and variable between cases. ${ }^{2}$ Common symptoms include fever, cough and malaise. Although it is considered a respiratory tract infection, however, some patients may initially attend with diarrhea and nausea a few days prior to fever. ${ }^{3,4}$ Moreover, asymptomatic carriers with radiological features in their computed tomography (CT) of the chest could exist and transmit infection. ${ }^{5}$ The Royal College of Surgeons (RCS) recommended to perform chest (CT) scan for any patient presenting to the emergency department (ED) with an acute abdomen that will necessitate an abdominal CT. ${ }^{6}$ Thin-slice chest CT plays a vital role in early detection, observation, and disease evaluation. ${ }^{7}$ To date, CT findings have been recommended as initial evidence for clinical diagnosis of COVID-19 in China. ${ }^{8}$ In this study we looked at the actual benefit of adding a routine chest CT with the CT KUB as an investigation in patients presenting to the ED with symptoms suggestive of renal colic in a UK district general hospital, and compared it with a group of patients with similar presentation in the pre-COVID-19 pandemic.

\section{METHODS}

We retrospectively extracted data at the peak of the pandemic in April 2020 for a group of 100 patients who presented to the ED in our hospital with suspected renal colic (Group 1); they all had the RCS-COVID recommended extended non-contrast CT scan (including chest and abdomen) and compared to a cohort of 100 patients with similar presentation in the pre-COVID-19 pandemic (Group 2); performed randomly selected from patients presenting between August and October 2019. The data in Group 2 was previously collected as part of the Royal College of Radiologists (RCR) audit to optimize CT KUB imaging in investigation of renal colic.

The following data were recorded and compared for both groups:

- Patients demographics

- Number of CT slices above the upper pole of the kidney. (Extra CT slices in comparison to routine scan)

- Vertebral level at the end of the scan

- Dose length product (DLP)

- Stone identification and stone size

- Any other incidental findings

- The Wuhan coronavirus swab test was performed in cases with chest CT confirming GGO or according to any other suspected features

\section{Statistics}

All the extracted data was analyzed using IPSS IBM Software version 17. This included doing Tindependent tests and chi square to compare between different parameters. $\mathrm{P}$ value of 0.005 was agreed to be of significant.

\section{RESULTS}

In the whole cohort of patients' age varied from 15 to 91 years with an average of 48 years in Group 1, while it ranged from 19 to 85 years with an average of 50 years in Group 2. Males had 55\% share in Group 1 and it was almost the same percentage 62\%in Group 2.

CT KUB confirmed urolithiasis in 51\% (51/100) of patients in Group 1 with an average stone size of 5.2 $\mathrm{mm}( \pm 4.6)$, while $50 \%(50 / 100)$ of Group 2 patients had urinary stones with average size $4.4 \mathrm{~mm}( \pm 2.1)$. Other incidental findings rather than stones blamed for patient's presentation with abdominal symptoms was recorded in both groups including diverticulitis, hepatic and gynecological pathologies, these results are summarized in (Table 1).

The vertebral level provided a useful coordinate system to calculate measurements for CT KUB with a chest $\mathrm{CT}$ included, and they were used to estimate the amount of extra amount of radiation received by patients. The RCR has accepted CT KUB should be 
TABLE 1 Other Incidental findings in the CT KUB Rather Than Stones

\begin{tabular}{|l|c|c|c|c|}
\hline $\begin{array}{l}\text { Other findings in } \\
\text { the CT KUB }\end{array}$ & \multicolumn{2}{|c|}{ Group 1 } & \multicolumn{2}{c|}{ Group 2 } \\
\hline & Frequency & Percentage \% & Frequency & Percentage \% \\
\hline Spines (Ankylosis & 2 & 2 & 1 & 1 \\
spondylitis) & 2 & 2 & 1 & 2 \\
Ovarian cyst & 4 & 45.9 & 6 & 6 \\
Cholecystitis & 3 & 3 & 1 & 2 \\
Diverticulitis & 1 & 12 & & \\
Endometrial cancer & 1 & 1 & 1 & 2 \\
Free fluid & 1 & 1 & 1 & 2 \\
Renal mass & 1 & 1 & 4 & 1 \\
Lymphadenopath & 1 & 1 & 1 & 1 \\
y & 1 & 1 & 2 & \\
Lung opacity & & & 1 & \\
Renal cyst & & & & \\
Fecal loaded & & & & \\
colon & & & & \\
Fatty liver & & & & \\
Splenic lesion & & & & \\
AAA & & & & \\
\hline
\end{tabular}

$A A A=$ abdominal aortic aneurysm.

limited between the level of the upper border of the highest kidney (T10 -T12) to the symphysis pubis. ${ }^{9} \mathrm{~A}$ total of 28\% (28100) of patients in Group 1 had their scans ending between the T1-T2 vertebra, while in Group 2 the scan ended at the T11 level as the most common vertebra $37.3 \%(37 / 100)(\mathrm{p}=0.001)$. The starting point of both scans was marked at the lesser trochanter by $49 \%(49 / 100)$ and $64 \%(64 / 100)$ in Group 1 and Group 2 respectively. ( $\mathrm{p}=0.500)$ (Tables 2 and 3$)$.

Although that upper pole of the highest kidney is recommended as the reference point for the upper limit of CT-KUB by the RCR, however it was noticed that even in Group 2 that did not include the chest CT there was still extra slices recorded above this reference point, therefore there is a risk of associated unnecessary radiation exposure.

Extra CT slices in both groups has been recorded and compared confirming average of $136(1 \mathrm{~mm})$ slices $( \pm 113)$ in Group 1 comparing to average of 36 extra slices $( \pm 26)(\mathrm{p}=0.001)$.
As regards DLP which is a measure of CT tube radiation output and the exposure for each patient to radiation. The DLP is given the radiation in units of milli-gray times the scan length in centimetre. In Group 1, we discovered that the minimum DLP exposure in our patients was $131 \mathrm{mGy} * \mathrm{~cm}$ and maximum of 1441 $\mathrm{mGy} * \mathrm{~cm}$ with an average of $526 \mathrm{mGy} * \mathrm{~cm}( \pm 322.5)$, however, in Group 2 the minimum was 127 and the maximum was 638 with an average of $289 \mathrm{mGy}^{*} \mathrm{~cm}$ $( \pm 106.6)(\mathrm{P}=0.001)$.

COVID-19 pneumonia has various chest CT findings. In our study, Group 1 had 28 patients who had full chest CT (up to T1 level) included during their CT KUB scan and 49 patients had lung base (up to T7 level) included during their CT KUB. Only 9 patients from the 77 patients demonstrated GGO.

In the same Group 9 patients were suspected from the GGO or other manifestations and tested with nasopharyngeal (NP) and oropharyngeal (OP) swabs yet all of them came back negative. 
TABLE 2 Vertebral Level at Which the Scan Ended

\begin{tabular}{|l|c|c|c|c|}
\hline Vertebral Levels & \multicolumn{2}{|c|}{ Group 1 } & \multicolumn{2}{c|}{ Group 2 } \\
\hline & Frequency & Percentage \% & Frequency & Percentage \% \\
\hline T1 & 24 & 24 & & \\
T2 & 4 & 4 & & \\
T6 & 3 & 3 & & \\
T7 & 10 & 10 & 1 & 1 \\
T8 & 18 & 18 & 3 & 3 \\
T9 & 7 & 7 & 8 & 26 \\
T10 & 11 & 11 & 26 & 37 \\
T11 & 15 & 15 & 37 & 21 \\
T12 & 8 & 8 & 21 & 4 \\
L1 & & & 4 & \\
\hline
\end{tabular}

TABLE 3 Lower Level at Which the Scan Started

\begin{tabular}{|l|c|c|c|c|}
\hline Lower Level & \multicolumn{2}{|c|}{ Group 1 } & \multicolumn{2}{c|}{ Group 2 } \\
\hline & Frequency & Percentage \% & Frequency & Percentage \% \\
\hline Greater trochanter & 30 & 30 & 5 & 5 \\
Lesser trochanter & 49 & 49 & 64 & 64 \\
Symphysis pubis & 21 & 21 & 10 & 10 \\
Proximal femur & & & 20 & 20 \\
Mid femur & & & 1 & 1 \\
\hline
\end{tabular}

\section{DISCUSSION}

In this study, patients who presented with acute renal colic were investigated with a CT KUB as part of their diagnostic investigation in accordance with the British Association of Urology guidance. ${ }^{10}$ During the COVID-19 pandemic they also had a chest $\mathrm{CT}$ as a standard scan for any patient presenting with abdominal symptoms. We evaluated the importance of performing a chest $\mathrm{CT}$ in emergency renal colic during the peak of the pandemic.

In our study, 77 patients had a chest CT included during their CT KUB scan. Only 9 patients (11.6\%) from the 77 patients demonstrated GGO which is very low output in relation to the number of the scans done and the radiation exposure needed. The common chest CT findings for COVID-19 are multifocal bilateral GGOs with patchy consolidations, with prominent peripheral subpleural distribution and a preference for a posterior location and a lower lobe predilection being the most typical chest CT findings that suggest the COVID-19 infection. ${ }^{11-13}$ However, controversial opinions regarding GGO were illustrated. Bernheim et al in 121 patients demonstrated that GGO were not detected in 56\% (20/36) of early phase patients who were COVID-19 positive and suggested that chest $\mathrm{CT}$ has limited sensitivity and negative predictive value early after symptom onset, and is thereby unlikely a reliable standalone tool to rule out COVID-19 infection. ${ }^{8}$ However, this is somewhat at odds with S. Inui et al who reported in 104 cases, that $76(73 \%)$ were asymptomatic and the chest CT findings showed a predominance of GGO over con-solidation in $83 \%$ of the asymptomatic patients on the cruise ship Diamond Princess. $^{14}$

The diagnostic importance of the chest CT was reported by Fang $\mathrm{Y}$ et al who concluded that the procedure had a sensitivity of $98 \%$ for early presentation of COVID-19 compared with $71 \%$ using the RT-PCR assay. ${ }^{3}$ Careful evaluation of the lung bases for GGO may lead to early diagnosis of COVID-19 before the presence of respiratory symptoms. ${ }^{15}$ 
T. Ai et al had 1014 patients, 59\% (601/1014) had positive RT-PCR results, and $88 \%(888 / 1014)$ had positive chest CT scans. The sensitivity of chest CT in suggesting COVID-19 was 97\% (95\% CI, 95-98\%, $580 / 601$ patients) based on positive RT-PCR results. In patients with negative RT-PCR results, 75\% (308/413) had positive chest CT findings; of $308,48 \%$ were considered as highly likely cases, with $33 \%$ as probable cases. The study concluded that chest CT may be considered as a primary tool for the current COVID-19 detection in epidemic area due to its high sensitivity ${ }^{16}$ However, the high diagnostic value of the chest CT was not apparent in our cohort. The up-todate National DRL (Diagnostic Reference Levels) for CT KUB is a DLP of $440 \mathrm{mGy}{ }^{*} \mathrm{~cm}$ or less. ${ }^{17}$ On the other hand, The UK National Dose Reference Level for CT scans of the abdomen and pelvis for KUB examinations assessing stones/colic is $745 \mathrm{mGy}^{*} \mathrm{~cm} .{ }^{18}$ In our cohort, the average DLP was of $526 \mathrm{mGy} * \mathrm{~cm}$ $( \pm 322.5)$. Therefore, we could say that we are in the grey zone between both national guidance. If this value was compared to Group 2 DLP $289 \mathrm{mGy}^{*} \mathrm{~cm}$ $( \pm 106.6)(\mathrm{p}=0.001)$, it will show the magnitude of the radiation for which those patients in Group 1 have been exposed to without providing much in the way of additional diagnostic value.

Extrapulmonary manifestations of COVID-19 in the form of abdominal pain is a real challenge for physicians since there are so many differential diagnoses. Although COVID-19 infections may present as an acute abdominal pain, Shihua Luo et al reported that 183/1141 (16\%) of confirmed COVID-19 patients had presented with gastrointestinal symptoms only. 45/183 (25\%) of the patients presented with abdominal pain. ${ }^{19}$

In our study, $49 \%$ of the patients attended with abdominal complain who underwent CT KUB were confirmed to have stones, while the rest of them had other finding rather than stones to explain their abdominal symptoms. However, no one in our patients was diagnosed with COVID-19 during or after his admission to the ED.

RT-PCR is currently considered as the gold standard diagnostic method for COVID-19 nasopharyngeal (NP) and oropharyngeal (OP) swabs were obtained from $(9 / 100)$ patients in our cohort as they were suspected COVID-19 and tested by (RT-PCR) came back negative. RT-PCR could come back negative be due to multiple factors including improper sampling and low viral load. ${ }^{3}$ There are many potential factors which may have contributed to these different results. Firstly, our patient cohort is limited to urological patients only. Secondly, due to the lockdown in the UK, the awareness of COVID-19 within the medical and general population had increased significantly, with social distancing in the community already in place by this stage which may have decreased the incidence of infection within the community. Further studies in the utility of the chest $\mathrm{CT}$ with any abdominal $\mathrm{CT}$ in the COVID-19 era is recommended to be compared with our results to assess the difference for other abdominal complains rather than renal colic.

\section{CONCLUSION}

Whilst $9 \%$ of patients in our study were diagnosed with chest CT changes suggestive of COVID, in no cases did this impact on their subsequent management or outcome. Furthermore, despite RCS CT-KUB recommendations our results are confirming a high radiation exposure with no sound clinical justification in asymptomatic cases.

Although COVID-19 has no specific urological manifestations, it is important that urologists must be alert to potential asymptomatic infection in patients presenting with urological pathology.

We would therefore like to conclude that the routine inclusion of a chest $\mathrm{CT}$ in the evaluation of men with suspected colic undergoing CT KUB is of no additional benefit; furthermore, it results in a significant escalation in the radiation dose delivered to this patient group.

Given the predilection for the GGOs to appear in an inferior and posterior location a standard CT KUB is likely to identify these changes without the need for additional imaging.

\section{DECLARATIONS OF INTEREST}

None.

\section{REFERENCES}

1. Vu D, Ruggiero M, Choi WS, Masri D, et al. Three unsuspected CT diagnoses of COVID-19. Emerg Radiol 2020; $1-4$. 
2. Huang C, Wang Y, Li X, Ren L, et al. Clinical features of patients infected with 2019 novel coronavirus in Wuhan, China. Lancet 2020;395(10223):497-506.

3. Fang Y, Zhang H, Xie J, Lin M, et al. Sensitivity of chest CT for COVID-19: Comparison to RT-PCR. Radiology 2020;19:200432. doi: 10.1148/radiol.2020200432. Online ahead of print.

4. Shi H, Han X, Jiang N, Cao Y, et al. Radiological findings from 81 patients with COVID-19 pneumonia in Wuhan, China: a descriptive study. Lancet Infect Dis. 2020;20(4):425-34.

5. Hu Z, Song C, Xu C, Jin G, et al. Clinical characteristics of 24 asymptomatic infections with COVID-19 screened among close contacts in Nanjing, China. Sci China Life Sci. 2020;May;63(5):706-11. doi: 10.1007/ s11427-020-1661-4. Epub 2020 Mar 4.

6. Guidance N, Ct C, General U, Guidance S. Guidance for Pre-Operative Chest CT Imaging for Elective Cancer Surgery During the COVID-19 Pandemic. Available at: https://www.rcseng.ac.uk/coronavirus/ preoperative-chest-ct-imaging-guidance/

7. Zu ZY, Jiang M Di, Xu PP, Chen W, et al. Coronavirus Disease 2019 (COVID-19): A Perspective from China. Radiology. 2020;2019:200490.

8. Bernheim A, Mei X, Huang M, Yang Y, et al. Chest CT Findings in Coronavirus Disease-19 (COVID-19): Relationship to Duration of Infection. Radiology 2020;295:3. Available at: https://pubs.rsna.org/doi/10.1148/ radiol.2020200463

9. Golding RP. Fundamentals of Body CT 4th Edition. Radiology. 2014.

10. Tsiotras A, Smith RD, Pearce I, O’Flynn K, et al. British Association of Urological Surgeons standards for management of acute ureteric colic. J Clin Urol 2018;11(1):58-61.

11. Kanne JP. Chest CT findings in 2019 novel coronavirus (2019-NCoV) infections from Wuhan, China: Key points for the radiologist. Radiology 2020;295:1.
12. Kim H. Outbreak of novel coronavirus (COVID-19): What is the role of radiologists? Eur Radiol 2020;30:3266-67.

13. Lee KS. Pneumonia associated with 2019 novel coronavirus: Can computed tomographic findings help predict the prognosis of the disease? Korean J Radiol 2020 Mar;21(3):257-58.

14. Inui S, Fujikawa A, Jitsu M, Kunishima N, et al. Chest CT Findings in Cases from the Cruise Ship Diamond Princess with Coronavirus Disease 2019 (COVID-19). Radiol Cardiothorac Imaging 2020; Available at: https:// doi.org/10.1148/ryct.2020200110

15. Siegel A, Chang PJ, Jarou ZJ, Paushter DM, et al. Lung base findings of coronavirus disease (COVID-19) on abdominal CT in patients with predominant gastrointestinal symptoms. Am J Roentgenol. 2020; 1-3. 10.2214/AJR.20.23232.

16. Ai T, Yang Z, Hou H, Zhan C, et al. Correlation of chest CT and RT-PCR testing in coronavirus disease 2019 (COVID-19) in China: A report of 1014 cases. Radiology 2020;https://doi.org/10.1148/radiol.2020200642

17. Shrimpton PC, Hillier MC, Meeson S, Golding SJ. Doses from Computed Tomography ( CT ) Examinations in the UK - 2011 Review About Public Health England. Pub Health Engl 2014. Available at: https://assets.publishing. service.gov.uk/government/uploads/system/uploads/ attachment_data/file/349188/PHE_CRCE_013.pdf

18. McLaughlin PD, Murphy KP, Hayes SA, Carey K, et al. Non-contrast CT at comparable dose to an abdominal radiograph in patients with acute renal colic; impact of iterative reconstruction on image quality and diagnostic performance. Insights Imaging 2014;Apr;5(2):217-30. doi: 10.1007/s13244-014-0310-z. Epub 2014 Feb 7.

19. Luo S, Zhang X, Xu H. Don't overlook digestive symptoms in patients with 2019 novel coronavirus disease (COVID-19). Clin Gastroenterol Hepatol 2020;Jun;18(7):1636-37. 